\title{
Observation-Informed Generalized Hybrid Error Covariance Models
}

\author{
ElizABETH A. SATTERFIELD AND DANIEl HodysS \\ Naval Research Laboratory, Monterey, California \\ DAVID D. KUHL \\ Naval Research Laboratory, Washington, D.C. \\ CRAIG H. BISHOP \\ Naval Research Laboratory, Monterey, California
}

(Manuscript received 17 January 2018, in final form 24 July 2018)

\begin{abstract}
Because of imperfections in ensemble data assimilation schemes, one cannot assume that the ensemblederived covariance matrix is equal to the true error covariance matrix. Here, we describe a simple and intuitively compelling method to fit calibration functions of the ensemble sample variance to the mean of the distribution of true error variances, given an ensemble estimate. We demonstrate that the use of such calibration functions is consistent with theory showing that, when sampling error in the prior variance estimate is considered, the gain that minimizes the posterior error variance uses the expected true prior variance, given an ensemble sample variance. Once the calibration function has been fitted, it can be combined with ensemble-based and climatologically based error correlation information to obtain a generalized hybrid error covariance model. When the calibration function is chosen to be a linear function of the ensemble variance, the generalized hybrid error covariance model is the widely used linear hybrid consisting of a weighted sum of a climatological and an ensemble-based forecast error covariance matrix. However, when the calibration function is chosen to be, say, a cubic function of the ensemble sample variance, the generalized hybrid error covariance model is a nonlinear function of the ensemble estimate. We consider idealized univariate data assimilation and multivariate cycling ensemble data assimilation to demonstrate that the generalized hybrid error covariance model closely approximates the optimal weights found through computationally expensive tuning in the linear case and, in the nonlinear case, outperforms any plausible linear model.
\end{abstract}

\section{Introduction}

In a chaotic system like the atmosphere, the true error covariance of short-term forecasts is highly flow dependent. Limited ensemble size and imperfections in methods used for initial condition and model error perturbations mean that ensemble covariances are inevitably inaccurate. Hence, there is a distribution of true covariances, given an imperfect ensemble covariance. The imperfections of ensemble-based estimates of the forecast error covariance are well recognized. Researchers such as Anderson (2007), Furrer and Bengtsson (2007), Sacher and Bartello (2008), Bocquet (2011), and Ménétrier et al. (2015) have suggested methods to

Corresponding author: Elizabeth Satterfield, elizabeth.satterfield@ nrlmry.navy.mil ameliorate the effect of these imperfections on the analysis correction and/or the covariance of the prior ensemble produced by ensemble data assimilation schemes. Bishop and Satterfield (2013) and Bishop et al. (2013) showed that if 1) the climatological probability density function (PDF) of true error variances takes the form of an inverse-gamma PDF and 2) the PDF of ensemble variances, given a true error variance, is given by a gamma PDF, then the PDF of true error variances, given an ensemble variance, is also an inverse-gamma PDF. In this case, the mean or minimum error variance estimate of the true error variance is a weighted linear combination of the mean climatological error variance and an imperfect ensemble variance. In other words, it is a linear hybrid.

Many practical tests (Etherton and Bishop 2004; Wang et al. 2008a,b, 2013; Yaremchuk et al. 2011; 
Clayton et al. 2013; Kuhl et al. 2013; Ménétrier and Auligné 2015) suggest that hybrid error covariance models (Hamill and Snyder 2000; Lorenc 2003) yield superior data assimilation performance to either just localized ensemble covariances (Hamill et al. 2001) or quasi-static climatological error covariance models. Ménétrier and Auligné (2015) outline a variational approach to optimize localization and hybridization simultaneously and further show that localizedhybridized covariances are more accurate than their localized-only counterparts, whatever the static covariance matrix specified. Their method was based on considering sampling error only and adjusting parameters to minimize the difference with the covariance matrix obtained in the limit of a large ensemble size.

A primary aim of this paper is to investigate the extent to which optimal hybridization parameters can be derived from an archive of (observation minus forecast, ensemble variance) pairs generated by a single long run of a hybrid data assimilation scheme. Here, we derive a simple and intuitively compelling formula to obtain the mean of this distribution of true error variances, given an ensemble variance. Such an approach is attractive because of its simplicity and flexibility, benefits from the use of observational information, and has the potential to greatly reduce the amount of time required to tune hybrid error covariance models.

The rest of this paper is organized as follows. Section 2 provides the detailed Bayesian development required to rigorously understand how hybrid covariance models relate to data assimilation. The application and verification of this theory in two univariate model examples are presented in section 3 . The development and application of a method applicable to numerical models are presented with a simple model case using the "Lorenz ' 96 " model in section 4 . The conclusions are presented in section 5 .

\section{Theory}

The goal of ensemble data assimilation is to obtain a posterior distribution for the state variable $x$, given the ensemble-based estimate $\sigma_{e}^{2}$ of the true prior variance $\sigma_{t}^{2}$ and the observation $y$ of the state $x$. To this end, we will first define the Bayesian formulation for this problem. Next, we will expand on the work of Bishop and Satterfield (2013) to define the prior variance conditioned on the ensemble variance and show that the mean of that distribution results in a hybrid covariance model. Last, we show the relationship between this result and the optimal Kalman gain.

\section{a. Bayesian formulation}

We would like to perform data assimilation in the situation in which the sample estimate of the prior variance is known but the true prior variance is unknown. To this end, we formulate the problem using the language of Bayesian statistics. Using the chain rule of probability, we write the joint PDF governing the relevant variables in two different ways:

$$
\begin{aligned}
p\left(x, y, \sigma_{t}^{2}, \sigma_{e}^{2}\right) & =p(x) p\left(\sigma_{e}^{2} \mid x\right) p\left(y \mid x, \sigma_{e}^{2}\right) p\left(\sigma_{t}^{2} \mid y, \sigma_{e}^{2}, x\right) \\
& =p\left(\sigma_{e}^{2}\right) p\left(y \mid \sigma_{e}^{2}\right) p\left(\sigma_{t}^{2} \mid y, \sigma_{e}^{2}\right) p\left(x \mid \sigma_{t}^{2}, \sigma_{e}^{2}, y\right) .
\end{aligned}
$$

We emphasize that the densities in (1) use the standard notation that the conditioning on prior observations from previous cycles is suppressed.

In solving for $p\left(x \mid \sigma_{t}^{2}, \sigma_{e}^{2}, y\right)$ in (1), we obtain a formula for the posterior distribution for the state variable $x$, namely,

$$
p\left(x \mid \sigma_{t}^{2}, \sigma_{e}^{2}, y\right)=\frac{p(x) p\left(\sigma_{e}^{2} \mid x\right) p\left(y \mid x, \sigma_{e}^{2}\right) p\left(\sigma_{t}^{2} \mid y, \sigma_{e}^{2}, x\right)}{p\left(\sigma_{e}^{2}\right) p\left(y \mid \sigma_{e}^{2}\right) p\left(\sigma_{t}^{2} \mid y, \sigma_{e}^{2}\right)} .
$$

Because we do not know the true prior variance $\sigma_{t}^{2}$, we must marginalize with respect to that random variable to make use of this theory. In other words, what we really need is $p\left(x \mid \sigma_{e}^{2}, y\right)$. To this end, we note that

$$
p\left(x \mid \sigma_{e}^{2}, y\right)=\int_{0}^{\infty} p\left(x \mid \sigma_{t}^{2}, \sigma_{e}^{2}, y\right) p\left(\sigma_{t}^{2} \mid y, \sigma_{e}^{2}\right) d \sigma_{t}^{2},
$$

which simplifies to

$$
p\left(x \mid \sigma_{e}^{2}, y\right)=\frac{p(x) p\left(\sigma_{e}^{2} \mid x\right) p\left(y \mid x, \sigma_{e}^{2}\right)}{p\left(\sigma_{e}^{2}\right) p\left(y \mid \sigma_{e}^{2}\right)} .
$$

Note further that another application of the chain rule for the prior reveals that

$$
p\left(x \mid \sigma_{e}^{2}\right)=\frac{p(x) p\left(\sigma_{e}^{2} \mid x\right)}{p\left(\sigma_{e}^{2}\right)} .
$$

By using (5) in (4), we obtain

$$
p\left(x \mid \sigma_{e}^{2}, y\right)=\frac{p\left(y \mid x, \sigma_{e}^{2}\right)}{p\left(y \mid \sigma_{e}^{2}\right)} p\left(x \mid \sigma_{e}^{2}\right),
$$

where the prior is $p\left(x \mid \sigma_{e}^{2}\right)$, the new observation likelihood is $p\left(y \mid x, \sigma_{e}^{2}\right)$, and the sought-after posterior is $p\left(x \mid \sigma_{e}^{2}, y\right)$.

\section{b. Application to sampling error}

To proceed, we assume that there is no model error and that the only issue with the ensemble variance estimate is sampling statistics. In this case, the observation likelihood in (6) simplifies to 


$$
p\left(y \mid x, \sigma_{e}^{2}\right)=p(y \mid x) .
$$

By contrast, note that, when there is model error (such as representation error) and the state variable $x$ is no longer a random draw from the true distribution, the simplification in (7) is not generally valid. In any event, when (7) holds, then (6) simplifies to

$$
p\left(x \mid \sigma_{e}^{2}, y\right)=\frac{p(y \mid x)}{p\left(y \mid \sigma_{e}^{2}\right)} p\left(x \mid \sigma_{e}^{2}\right),
$$

which shows that the impact on the state variable from the sampling error only comes in through the prior. In this section, we restrict attention to the case in which the true prior for the state variable is Gaussian:

$$
p\left(x \mid \sigma_{t}^{2}\right)=\frac{1}{\sqrt{2 \pi \sigma_{t}^{2}}} \exp \left[-\frac{1}{2} \frac{(x-\bar{x})^{2}}{\sigma_{t}^{2}}\right],
$$

where $\bar{x}$ is the true prior mean. As discussed in many places (e.g., Hodyss et al. 2016), when the true prior for the state variable is Gaussian and is given by (8), sampling statistics have the property that the sample variance $\sigma_{e}^{2}$ may be written as

$$
a=\left(N_{e}-1\right) \frac{\sigma_{e}^{2}}{\sigma_{t}^{2}}
$$

where $a$ is a random variable that is $\chi^{2}$ with $N_{e}-1$ degrees of freedom and $N_{e}$ is the sample size.

Given that the ensemble provides an imperfect estimate of the true prior error variance, we would like to know the distribution of true variances, given an ensemble estimate $p\left(\sigma_{t}^{2} \mid \sigma_{e}^{2}\right)$. As discussed by Bishop and Satterfield (2013), when $p\left(\sigma_{e}^{2} \mid \sigma_{t}^{2}\right)$ is a $\chi^{2}$ (gamma) PDF and the climatological PDF of true error variances $p\left(\sigma_{t}^{2}\right)$ is an inverse-gamma PDF (with shape and scale parameters $\alpha_{t}$ and $\left.\beta_{t}\right)$, this implies that $p\left(\sigma_{t}^{2} \mid \sigma_{e}^{2}\right)$ is also inverse gamma distributed. Under these assumptions, we have

$$
p\left(\sigma_{t}^{2} \mid \sigma_{e}^{2}\right)=\frac{\beta^{\alpha}}{\Gamma(\alpha)}\left(\frac{\sigma_{e}^{2}}{\sigma_{t}^{2}}\right)^{\alpha+1} \exp \left(-\frac{\beta}{\sigma_{t}^{2}}\right),
$$

where $\Gamma$ is the gamma function and $\alpha$ and $\beta$ are the shape and scale parameters:

$$
\begin{aligned}
& \alpha=\alpha_{t}+\frac{N_{e}-1}{2} \text { and } \\
& \beta=\beta_{t}+\frac{N_{e}-1}{2} \sigma_{e}^{2} .
\end{aligned}
$$

Consistent with Bishop and Satterfield (2013), this implies that the mean true prior variance conditioned on the ensemble variance is

$$
\left\langle\sigma_{t}^{2} \mid \sigma_{e}^{2}\right\rangle=\int_{0}^{\infty} \sigma_{t}^{2} p\left(\sigma_{t}^{2} \mid \sigma_{e}^{2}\right) d \sigma_{t}^{2}=\frac{\beta}{\alpha-1}=w_{e} \sigma_{e}^{2}+w_{c}\left\langle\sigma_{t}^{2}\right\rangle,
$$

where

$$
\begin{aligned}
w_{e} & =\frac{N_{e}-1}{2(\alpha-1)}, \\
w_{c} & =\frac{\alpha_{t}-1}{\alpha-1}, \text { and } \\
\left\langle\sigma_{t}^{2}\right\rangle & =\int_{0}^{\infty} \sigma_{t}^{2} p\left(\sigma_{t}^{2}\right) d \sigma_{t}^{2} .
\end{aligned}
$$

Hence, the mean of the true prior variance conditioned on the ensemble variance is a linear function of the ensemble variance under the assumptions of this section. Therefore, an estimate of the true variance is a "hybrid" that linearly weights the ensemble variance and the climatological variance. We note that our choice of an inverse gamma pdf $p\left(\sigma_{t}^{2}\right)$ for the climatological distribution of true error variances and a $\chi^{2}$ likelihood $p\left(\sigma_{e}^{2} \mid \sigma_{t}^{2}\right)$ results in a linear hybrid form consistent with (14) for the optimal error variance estimate. We note that Bocquet (2011) presents a very similar idea. In Bocquet (2011), accounting for sampling error is achieved by computing the prior PDF conditioned on the ensemble. This is consistent with (2), where we then marginalize with respect to the unknown true prior distribution of variances. In our case, instead of solving variationally, we show (see the appendix) that when sampling error is considered, the minimum posterior variance is achieved when the Kalman gain uses the mean of that conditional distribution (which is a known linear function in the gamma-inverse-gamma case). In what follows, we explore the relationship of mean true prior variances (14) to regression analysis and to data assimilation.

\section{c. Relationship to regression analysis}

It is well known that the mean of a conditional density [i.e., (14)] can be estimated using the techniques of regression. To that end, note that a linear regression model for the mean true prior variance, given an ensemble variance, would take the following form:

$$
P_{\mathrm{reg}}\left(\sigma_{e}^{2}\right)=\left\langle\sigma_{t}^{2}\right\rangle+G\left(\sigma_{e}^{2}-\left\langle\sigma_{e}^{2}\right\rangle\right)
$$

where

$$
\begin{gathered}
G=\frac{\operatorname{cov}\left(\sigma_{t}^{2}, \sigma_{e}^{2}\right)}{\operatorname{var}\left(\sigma_{e}^{2}\right)}, \\
\operatorname{cov}\left(\sigma_{t}^{2}, \sigma_{e}^{2}\right)=\left\langle\sigma_{t}^{4}\right\rangle-\left\langle\sigma_{t}^{2}\right\rangle^{2}=\operatorname{var}\left(\sigma_{t}^{2}\right), \quad \text { and }
\end{gathered}
$$




$$
\operatorname{var}\left(\sigma_{e}^{2}\right)=\frac{2}{N_{e}-1}\left\langle\sigma_{t}^{4}\right\rangle+\operatorname{var}\left(\sigma_{t}^{2}\right)
$$

with $\operatorname{cov}(x, y)=\langle(x-\langle x\rangle)(y-\langle y\rangle)\rangle$ and $\operatorname{var}(x)=$ $\left\langle(x-\langle x\rangle)^{2}\right\rangle$. If we use (10), (19), (20), and (21) along with the known properties of the inverse-gamma distribution, we may show that $w_{e}=G$ and $w_{c}=1-G$, which proves that the hybrid variance model in (14) is consistent with linear regression under the assumptions of section 2a. We demonstrate below that, when the climatological distribution is no longer inverse gamma, this relationship between $G$ and $w_{e}$ is broken.

In practice, we do not have available samples of $\sigma_{t}^{2}$ to use in a regression; therefore, we use squared innovations because it can be shown that

$$
\operatorname{cov}\left(\sigma_{t}^{2}, \sigma_{e}^{2}\right)=\operatorname{cov}\left(d^{2}, \sigma_{e}^{2}\right),
$$

where the innovation is written as $d=y-\bar{x}$. The relationship defined in (22) can be derived by noting that $y=x+\varepsilon_{o}$ and $x=\bar{x}+\sigma_{t} \zeta$, where $\varepsilon_{o}$ is a random variable with mean 0 and variance $\sigma_{o}^{2}$ and $\zeta$ is a random variable with mean 0 and variance 1 . Using these definitions for $y$ and $x$ in the innovation $d$ allows the proof for (22) to be completed.

\section{d. Relationship to data assimilation}

For applications to data assimilation, what we really want to know is how the prior state $x$ relates to the ensemble variance rather than the true variance. Note that in general this new prior is determined from

$$
p\left(x \mid \sigma_{e}^{2}\right)=\int_{0}^{\infty} p\left(x \mid \sigma_{t}^{2}\right) p\left(\sigma_{t}^{2} \mid \sigma_{e}^{2}\right) d \sigma_{t}^{2} .
$$

For the special case of an inverse-gamma climatological PDF of true error variances and a $\chi^{2}$ PDF of ensemble variances, given a true error variance, one can show that the structure of this prior is a kind of $t$ distribution of the form

$$
p\left(x \mid \sigma_{e}^{2}\right)=\frac{2^{\alpha} \beta^{\alpha}}{\sqrt{\pi}} \frac{\Gamma\left(\alpha+\frac{1}{2}\right)}{\Gamma(\alpha)}\left[\frac{1}{2 \beta+(x-\bar{x})^{2}}\right]^{\alpha+(1 / 2)} .
$$

The $t$ distribution in (24) is known to have fatter tails than a Gaussian and therefore shows that the uncertainty in the state $x$ for small ensemble size is a symmetric, but nonGaussian, distribution with fat tails.

A Kalman filter state estimate must make use of the variance of the prior. Again, we may calculate the variance of (24) to obtain

$$
P_{f}\left(\sigma_{e}^{2}\right)=\int_{-\infty}^{\infty}(x-\bar{x})^{2} p\left(x \mid \sigma_{e}^{2}\right) d x=\frac{\beta}{\alpha-1}=\left\langle\sigma_{t}^{2} \mid \sigma_{e}^{2}\right\rangle .
$$

Because (25) is equal to (14), we know that the prior variance about the true prior mean is also a "hybrid" that weights the ensemble prior variance and the climatological variance to find an estimate of the true prior variance. While we have now explicitly shown that the variance of the prior state $x$ is equal to the mean of the distribution of true prior variances, given a sample variance [e.g., (14)] for the specific case of sampling error for a Gaussian true prior [e.g., (9)], it is possible to show that this result holds for all priors by simply using (23) in (25) directly. Hence, the prior variance of a state variable $x$ when conditioned on the ensemble variance is always determined by the mean of the distribution of true variances, given a sample variance $P_{f}\left(\sigma_{e}^{2}\right)=\left\langle\sigma_{t}^{2} \mid \sigma_{e}^{2}\right\rangle$.

To formally relate this new form of prior to the Kalman filter state estimate, we need to minimize the average squared errors for the state variable $x$, and we emphasize that we do this under the assumptions of section $2 \mathrm{~b}$. To this end, we note that the average squared error in a Kalman state estimate is

$$
P_{a}\left(\sigma_{e}^{2}\right)=\int_{-\infty}^{\infty} \int_{-\infty}^{\infty} \int_{-\infty}^{\infty}\left(x-\bar{x}_{a}\right)^{2} p\left(x, y, \sigma_{t}^{2} \mid \sigma_{e}^{2}\right) d x d y d \sigma_{t}^{2},
$$

where

$$
\bar{x}_{e}\left(y, \sigma_{e}^{2}\right)=\bar{x}+G_{e}(y-\bar{x}) .
$$

We perform the standard operations to derive the Kalman gain $G_{e}$ that minimizes (26). Please see the appendix for the derivation. Briefly, by taking the derivative of (26) with respect to $G_{e}$, setting the result to zero, and subsequently solving for $G_{e}$, we obtain

$$
G_{e}=\frac{\left\langle\sigma_{t}^{2} \mid \sigma_{e}^{2}\right\rangle}{\left\langle\sigma_{t}^{2} \mid \sigma_{e}^{2}\right\rangle+\sigma_{o}^{2}} .
$$

Hence, we have now proven that the gain that minimizes the posterior variance uses the expected true prior variance, given an ensemble variance, as in (14). We note that this proof can be easily extended in a couple of ways. First, if we continue to make the assumption that the state estimate (27) makes use of the true prior mean, then one obtains (28) even for non-Gaussian true priors [i.e., replace (9) with a nonGaussian PDF]. Second, if we continue with the Gaussian assumption in (9) but replace the true prior mean with the sample prior mean in (27), we obtain a gain of the same form as (28) but for which the expected true prior variance, given an ensemble variance $\left\langle\sigma_{t}^{2} \mid \sigma_{e}^{2}\right\rangle$, is simply inflated by a factor $1+1 / N_{e}$ to 
account for the additional uncertainty from sampling error in the prior mean. However, because it can be shown that the sampling error in the prior mean and the sampling error in the prior variance are correlated for non-Gaussian (skewed) priors, extending the proof to non-Gaussian true priors (9), while also including sampling error in the prior mean in (27), is nontrivial and left to future work.

To illustrate the behavior of this new form of Kalman state estimate, we provide an example of data assimilation for the model of section $2 \mathrm{~b}$. We use a single scalar assimilation of a generic problem, where the typical assumptions are satisfied. The observations will be taken to have been drawn from a Gaussian observation likelihood with an observation error variance $\sigma_{o}^{2}$ equal to 1 . We plot (8) as a function of the observation $y$ for three different values of sample variance in Fig. 1. The parameters are set as $\bar{x}=0, \alpha_{t}=3, \beta_{t}=2$, and $N_{e}=5$. These values imply that the mean of the climatological PDF of prior error variance is $\left\langle\sigma_{t}^{2}\right\rangle=1$. Using these parameters, we plot the prior PDF in Fig. 1 for $\sigma_{e}^{2}$ equal to $0.1,1$, and 2, respectively. Along with the Bayesian posterior given by (8), in Fig. 1 we also plot (black line) the state estimate from a Kalman filter that makes use of the hybrid variance estimator in (14) and, equivalently, (18). This shows that the use of the hybrid variance estimator correctly aligns the Kalman state estimate along the true posterior mean in the high-probability region of the posterior PDF. Note, however, that for large values of the innovation (or the observation), the curvature of the posterior mean leads to large errors in the Kalman state estimate. At these extreme values of the innovation, the Kalman state estimate is too close to the prior mean and not close enough to the observed value: it does not draw the analysis close enough to the observation. This is a direct consequence of the fact that the Kalman filter mistakenly assumes that the forecast error variance is precisely known; it is not, and this uncertainty implies that the chances of the truth lying many standard deviations from the prior mean is increased.

\section{Univariate model experiments}

\section{a. Inverse gamma}

We may numerically verify the theory of section 2 for the case in which the climatological distribution of true variances is an inverse-gamma PDF and the PDF of ensemble variance given a true variance is a gamma PDF by letting the true state be a result of the stochastic process given by

$$
x=\bar{x}+\varepsilon^{f},
$$

where $\varepsilon^{f}$ is a draw from a normal Gaussian PDF with mean 0 and variance $\sigma_{t}^{2}$, with prior mean $\bar{x}$ equal to 1 . The observations will be taken to have a Gaussian observation likelihood with an observation error variance $\sigma_{o}^{2}$ equal to 1 . We employ $10^{7}$ trials. For each trial of this experiment, a sample truth will be created using (29) and random noise will be added to create an observation. The true forecast error variances will be drawn from an inverse-gamma PDF with $\alpha_{t}=3, \beta_{t}=2$, and $N_{e}=5$. The theory above [(15) and (16)] states that for this problem setup the weights are equal (i.e., $\left.w_{e}=w_{c}=0.5\right)$. We continue with the assumptions of this section and use the true prior mean; we will illustrate the modifications required when using the sample prior mean in the next section when using the Lorenz ' 96 model. Note that the use of the true prior mean here implies that prior inflation is not required because both the sample variance and the innovations are unbiased. To determine the linear hybrid regression model, we employ (18) by drawing ensembles from (29), calculating the sample variance, and regressing the sample variance against the true variance as discussed in section 2c. Of course, in a realistic application, we will not have available the true variance to regress; therefore, in the next section we discuss how to perform this regression using innovations. For now, however, we continue with the framework of section $2 \mathrm{c}$ to clearly verify the theory. The result of this regression finds $G=0.501$, which verifies that regression finds the appropriate weights. Figure 2 a plots this regression line as well as the true variances binned as a function of sample variances. The similarity of these two lines verifies (14) because it shows that the mean really is a straight line with the slope and intercept determined by $w_{e}$ and $w_{c}$, respectively.

For each trial of this experiment, we calculate three state estimates and measure their quality in terms of posterior mean square error (MSE). The first state estimate is to simply use the sample variance in the Kalman state estimate (i.e., the traditional ensemble Kalman filter) using $w_{e}=1$ and $w_{c}=0$, which obtains a posterior MSE of 0.50 . The second state estimate uses (27) with the hybrid variance model determined from (18), which obtains a posterior MSE of 0.472. The last state estimate we examine is determined by "brute force" tuning the weights of the hybrid covariance model to find the configuration with the smallest posterior MSE. Employing this procedure leads to $w_{e}=0.497$ and $w_{c}=$ 0.503 with a posterior MSE of 0.472 , in agreement with the linear hybrid variance model. While the improvement over simply using the sample variance is small, 

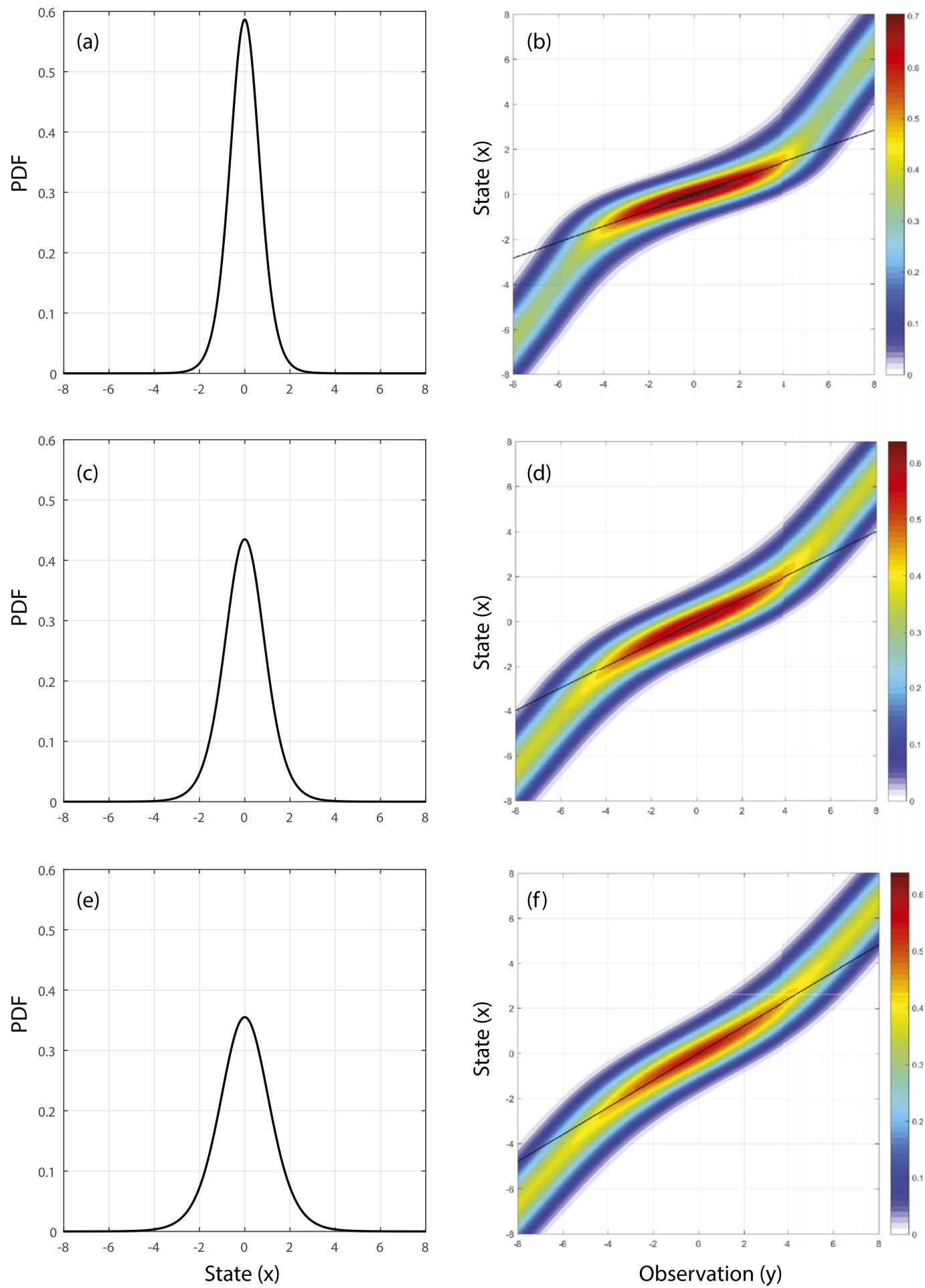

FIG. 1. The (left) prior and (right) posterior with ensemble variance values of (a),(b) 0.1, (c),(d) 1, and (e),(f) 2. The black line in the right panels shows the state estimate from a Kalman filter that makes use of the hybrid variance. All experiments assume $y \sim N\left(0, \sigma_{o}^{2}=1\right), \bar{x}=0, \alpha_{t}=3$, and $\beta_{t}=2$. 

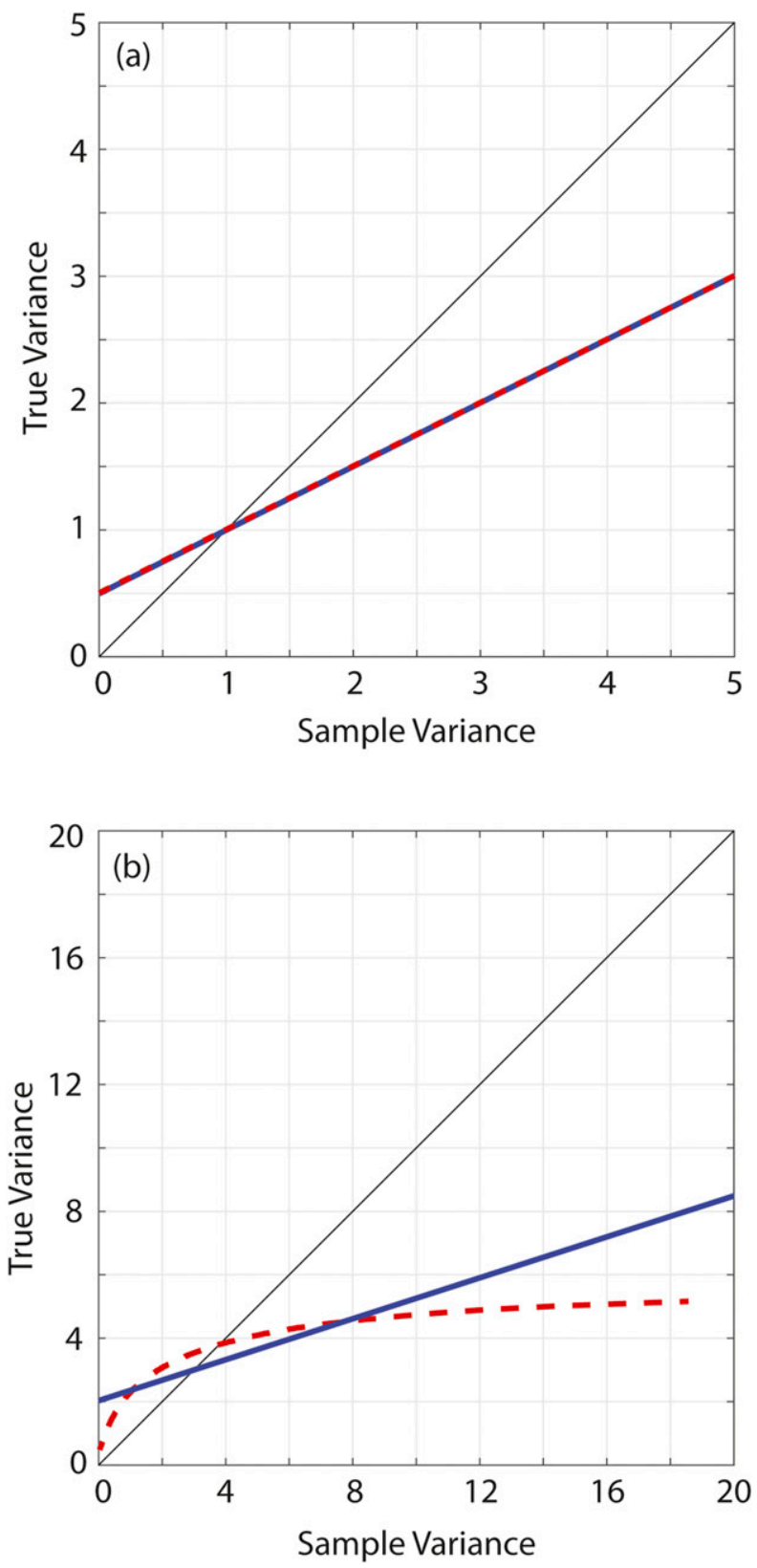

FIG. 2. True variance binned as a function of sample variance (red line) and linear regression of the true variance onto the sample variance (blue line) for cases in which the true variance is drawn from (a) an inverse-gamma distribution and (b) a uniform distribution.

it is nonetheless clear that the linear hybrid model is superior and that the weights determined from regression are in fact the ones that minimize the posterior MSE.

\section{b. Uniform}

The goal of this section is to show what happens when the climatological distribution of true variances differs from an inverse-gamma distribution. We make use of the same framework as section $2 \mathrm{~d}$. The only change we make is to create the random variable for the true variances using the following:

$$
\sigma_{t}^{2}=a+b u
$$

where $a=0.1, b=5.8$, and $u$ is a random variable drawn from the uniform distribution on 0 to 1 . We illustrate the structure of the prior and posterior distributions in this case in Fig. 3. Also, we again determine the linear hybrid regression model by drawing ensembles from (29), calculating the sample variance, and regressing the sample variance against the true variance. The linear regression line is plotted in Fig. 2b. Also shown in Fig. $2 b$ is the expected true variance as a function of the sample variance (red line), which is now a curve rather than a straight line, as in the previous section. This means that the linear estimate of the expected true variance by the linear hybrid is, in this case, incorrect.

To further illustrate this potential problem in the estimation, we again report the posterior MSE for three different experiments. For the first experiment, we simply use the sample variances that we obtain from an ensemble using $w_{e}=1$ and $w_{c}=0$, which obtains a posterior MSE of 0.796. Second, we employ regression to determine the weights for the hybrid variance model and find $G=0.322$, which when used as a hybrid variance model in a Kalman filter obtains a posterior MSE of 0.734 . Last, we again employ brute-force tuning of the weights of the hybrid covariance model to find the configuration with the smallest posterior MSE. Employing this procedure leads to $w_{e}=0.653$ and $w_{c}=$ 0.437 with a posterior MSE of 0.729 . Last, we could employ (27) with gain (28), for which we define $\left\langle\sigma_{t}^{2} \mid \sigma_{e}^{2}\right\rangle$ from the red curve in Fig. 2b. This configuration obtains a posterior MSE of 0.722 and, consistent with the theory of section $2 \mathrm{~d}$, provides the state estimate with the smallest posterior MSE.

Note that if regression had determined the correct weight on the ensemble variance then we would find that $G$ was equal to the $w_{e}$ obtained from brute-force tuning experiments. This is not the case for the climatological distribution of variances given by (30). Experiments (not shown here) with a variety of climatological distributions whose tails were narrower than the inversegamma distribution all found that $G<w_{e}$. Hence, we believe that this behavior is general and therefore that replacing the traditional linear hybrid variance model that cannot possibly account for this curvature with a generalized hybrid variance model that better accounts 

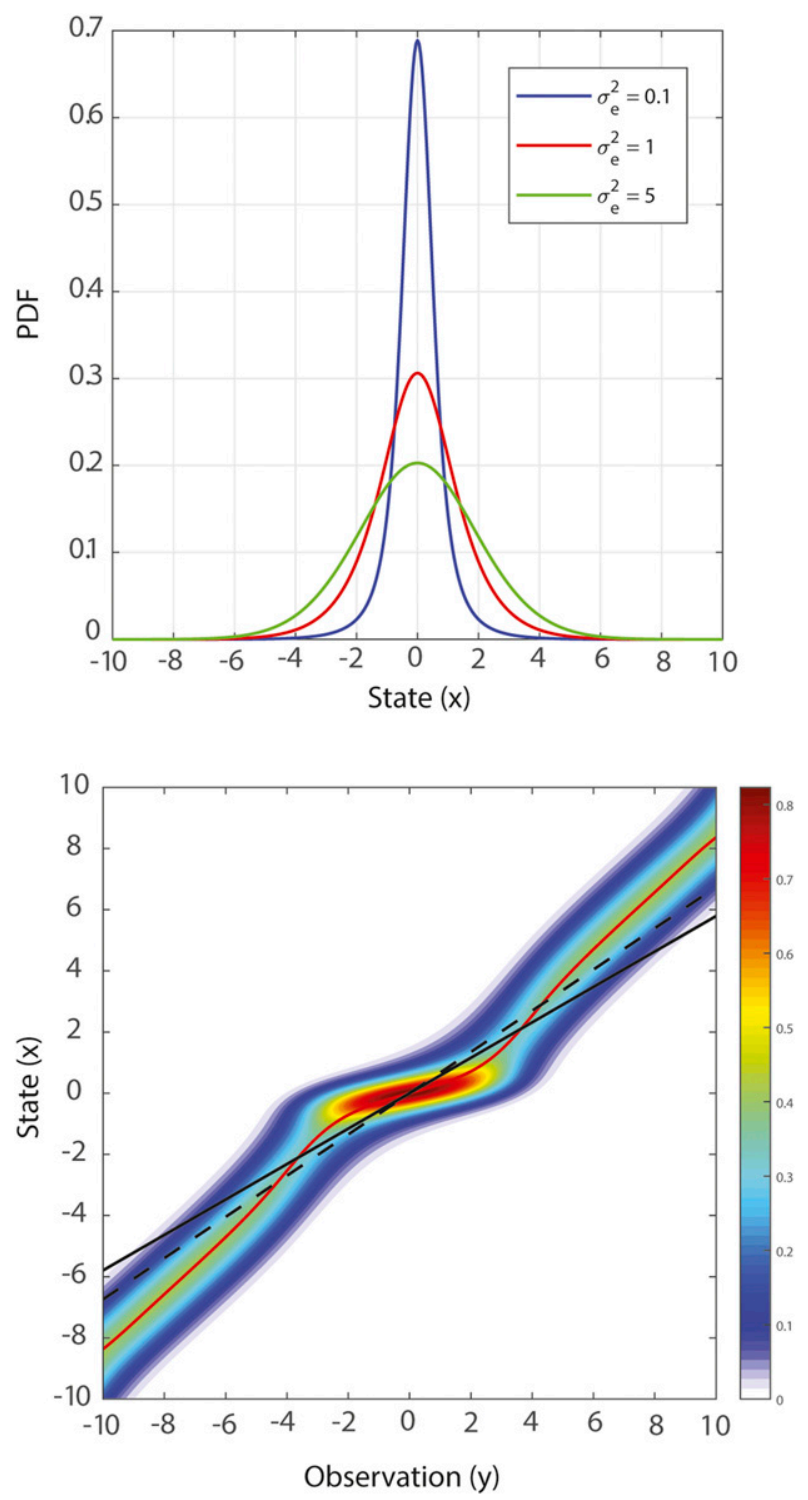

FIG. 3. As in Fig. 1, but for the case in which the true variances are drawn by a uniform distribution defined by $\sigma_{t}^{2}=a+b u$, where $a=0.1, b=5.8$, and $u$ is a random variable drawn from the uniform distribution on $0-1$. Also shown in the bottom panel is the linear regression (dashed black line) expected true variance as a function of the sample variance (red line), which is now a curve.

for this curvature will result in superior data assimilation performance.

\section{Lorenz'96 model experiments}

In this section, we explore the case in which, as in a real-world application, we do not know the climatological distribution of true error variances. To simplify things, we can control the observation error variance $\sigma_{o}^{2}$, observation density, and influence of model error. We will show that the $w_{e}$ value required to minimize the analysis error variance in a hybrid data assimilation system is underestimated by linear regression and that the true prior variance can be estimated as a higherorder polynomial function of the ensemble variance, consistent with section 2. We assess the optimal weighting of flow-dependent and static covariances using an implementation of the perturbed observations form of the ensemble Kalman filter on a 10-variable version of the Lorenz '96 model [Lorenz (1996); also see model 1 of Lorenz (2005)]. In what follows, we provide a brief description of this analysis/forecast system.

\section{a. Description of model and data assimilation}

We utilize the Lorenz model with a forcing term $(F=$ $8)$. We use a time step of $\Delta t=0.05$ and the fourth-order Runge-Kutta time integration scheme, while allowing for model spinup by disregarding the initial 240 time steps. We define the true state as a draw from this previously generated nature run. We note that the assimilation experiments are performed using the same setting as in the nature run. We calculate the background-error covariance from the ensemble mean as follows:

$$
\mathbf{P}^{f}=\frac{\mathbf{X}_{b} \mathbf{X}_{b}^{\mathrm{T}}}{N_{e}-1},
$$

where $\mathbf{X}_{b}$ denotes an $M \times N_{e}$ matrix of ensemble perturbations,

$$
\left\{\mathbf{X}_{b}=\mathbf{x}_{b i}-\overline{\mathbf{x}_{b}}: i=1, \ldots, k\right\},
$$

where $M$ is the model dimension and $N_{e}$ is the number of ensembles. Observations are created by perturbing the "truth" with observation error drawn from a zero-mean Gaussian distribution with variance $\sigma_{o}^{2}=0.5$ :

$$
\mathbf{y}=\mathbf{x}^{t}+\boldsymbol{\varepsilon}^{o}, \quad \boldsymbol{\varepsilon}^{o} \sim N\left(0, \sigma_{o}^{2}\right) .
$$

For the following experiments, we observe every grid point at each time step.

We assimilate observations using a stochastic update approach (Burgers et al. 1998) as follows:

$$
\begin{aligned}
\mathbf{K} & =\mathbf{P}^{f} \mathbf{H}^{\mathrm{T}}\left(\mathbf{H} \mathbf{P}^{f} \mathbf{H}^{\mathrm{T}}+\mathbf{R}\right)^{-1} \text { and } \\
\mathbf{P}^{a} & =\mathbf{P}^{f}-\mathbf{K} \mathbf{H} \mathbf{P}^{f},
\end{aligned}
$$

where $\mathbf{H}$ denotes the observation operator (which in this case is simply the identity matrix). We define

$$
\mathbf{T}=\mathbf{P}^{a} \mathbf{H}^{\mathrm{T}} \mathbf{R}^{-1} \text {. }
$$

We update the analysis ensemble following

$$
\mathbf{x}_{a i}=\mathbf{x}_{b i}+\mathbf{T}\left(\mathbf{y}_{i}-\mathbf{H} \mathbf{x}_{b i}\right),
$$


where $\mathbf{y}_{i}$ refers to the observation $\mathbf{y}$, defined in (33), perturbed with a random observation error. The analysis is defined as the ensemble mean:

$$
\overline{\mathbf{x}_{a}}=\frac{1}{N_{e}} \sum_{i=1}^{N_{e}} \mathbf{x}_{a i} .
$$

The ensemble forecasts are cycled to create the background-error covariance matrix for the next time step. Note that, because the covariance matrix of the $\mathbf{x}_{b i}$ is not equal to the hybrid covariance model, the resulting ensemble $\mathbf{x}_{a i}$ does not actually satisfy the posterior covariance equation in (34). To maintain consistency with standard ensemble generation methods, we nevertheless will use (36) and leave the derivation of an ensemble generation method that eliminates this issue to future work.

\section{b. Localization}

To avoid spurious correlations at longer range, we localize the ensemble-based covariance matrix by a Schur product with a correlation function as defined in Gaspari and Cohn (1999):

$$
\rho= \begin{cases}-\frac{1}{4}(|z| / c)^{5}+\frac{1}{2}(|z| / c)^{4}+\frac{5}{8}(|z| / c)^{3}-\frac{5}{3}(|z| / c)^{2}+1 & \text { for } \quad 0 \leq|z| \leq c \\ \frac{1}{12}(|z| / c)^{5}-\frac{1}{2}(|z| / c)^{4}+\frac{5}{8}(|z| / c)^{3}+\frac{5}{3}(|z| / c)^{2}-5(|z| / c)+4-\frac{2}{3}(c /|z|) & \text { for } \quad c \leq|z| \leq 2 c \\ 0 & \text { for } \quad 2 c \leq|z|\end{cases}
$$

where $z$ represents the Euclidean distance between grid points in physical space and $c$ is defined such that the correlation reduces from 1.0 to 0 at a distance of $2 c$. For the experiments that follow, we define $c=3$ on the basis of coarse tuning for the five-member ensemble case. All tuning experiments were integrated for $N_{t}=100000$ time steps and used a $100 \%$-ensemble-based error covariance matrix. The ensemble variance was coarsely tuned to match the mean squared error in the background forecast. We note that for the $N_{e}=10$ ensemble case the minimum RMSE was found when no localization was used. However, we chose to retain the $c=3$ for consistency and to prevent divergence for the $100 \%$-ensemble case.

\section{c. Experimental design and results}

We define the hybrid background-error covariance matrix following

$$
\mathbf{P}_{\text {hybrid }}^{f}=\left(\mathbf{g}_{\mathrm{ens}}\right)^{1 / 2}\left(\mathbf{C}_{\mathrm{ens}}\right) \mathbf{g}_{\mathrm{ens}}^{1 / 2}+\mathbf{g}_{\mathrm{clim}}^{1 / 2}\left(\mathbf{C}_{\mathrm{clim}}\right) \mathbf{g}_{\mathrm{clim}}^{1 / 2},
$$

where the correlation matrices are defined following

$$
\begin{aligned}
& \mathbf{C}_{\mathrm{ens}}=\mathbf{D}_{\mathrm{ens}}^{-1 / 2}\left(\rho \mathbf{P}_{\mathrm{ens}}^{f}\right) \mathbf{D}_{\mathrm{ens}}^{-1 / 2} \text { and } \\
& \mathbf{C}_{\mathrm{clim}}=\mathbf{D}_{\mathrm{clim}}^{-1 / 2}\left(\mathbf{P}_{\mathrm{clim}}^{f}\right) \mathbf{D}_{\mathrm{clim}}^{-1 / 2},
\end{aligned}
$$

and where the matrices $\mathbf{D}$ denote the diagonal matrices of variances. The diagonal matrix $\mathbf{g}_{\text {ens }}$ can be interpreted as the weight given to the flow-dependent covariances. When $\mathbf{g}_{\text {ens }}$ is defined by a linear function of the ensemble variances (e.g., $\mathbf{g}_{\text {ens }}=w_{e} \mathbf{D}_{\text {ens }}$ ), (38) can be written as a traditional linear hybrid, namely,

$$
\mathbf{P}_{\text {hybrid }}^{f}=w_{e} \mathbf{P}_{\text {ens }}^{f}+w_{c} \mathbf{P}_{\text {clim }}^{f} .
$$

We can extend this notation to the case in which the relationship between the true error variance and the sample variance is modeled by some nonlinear function, such as a higher-degree polynomial (as in Fig. 2b) $\mathbf{g}_{\text {ens }}=w_{e 1} \mathbf{D}_{\text {ens }}+w_{e 2} \mathbf{D}_{\text {ens }}^{\odot 2}+w_{e 3} \mathbf{D}_{\text {ens }}^{\odot 3}+\cdots$, where each element of $\mathbf{D}_{\text {ens }}^{\odot n}$ is the $n$th power of the corresponding element in the diagonal matrix of variances $\mathbf{D}_{\text {ens }}$.

For the ideal linear case, the climatological average of the ensemble variance $\left\langle\sigma_{e}^{2}\right\rangle$ and the climatological error variance $\sigma_{\text {clim }}^{2}$ are both approximately equal to the climatological average mean squared error $\left\langle\sigma_{f}^{2}\right\rangle$. In such a case, we expect that $w_{c}=1-w_{e}$, since in this case the coefficients are simply acting to weight the flow-dependent and static components rather than weight and also inflate the variance to account for unrepresented uncertainty.

In what follows, the static component of the backgrounderror covariance matrix $\mathbf{P}_{\text {clim }}^{f}$ is defined by collecting forecast errors from a $100 \%$-ensemble-based system for $M=10$ and taking the expectation (denoted $\langle$.$\rangle ) over N_{t}=100000$ time steps:

$$
\begin{aligned}
\left\{\mathbf{X}_{\mathrm{clim}}=\overline{\mathbf{x}_{b i}}-\mathbf{x}_{i}^{t}: i\right. & \left.=1, \ldots, N_{t}\right\}, \\
\mathbf{Z}_{\text {clim }}^{f} & =\frac{\mathbf{X}_{\text {clim }}}{\sqrt{N_{t}-1}}, \quad \text { and } \\
\mathbf{P}_{\text {clim }}^{f} & =\left\langle\mathbf{Z}_{\text {clim }}^{f} \mathbf{Z}_{\text {clim }}^{f}{ }^{\mathrm{T}}\right\rangle .
\end{aligned}
$$

Our experiments proceed as follows:

1) To start our experiments, we first run a fully ensemblebased $(\alpha=1)$ experiment, which is cycled for 100000 time steps (after disregarding the first 100 time steps to allow for spinup). This experiment also serves to 
generate a static $\mathbf{P}_{\text {clim }}^{f}$. This experiment uses a standard prior inflation (of $\rho=1.2$ ).

2) We iterate this $100 \%$-ensemble-based experiment 10 times, each time adjusting the prior ensemble variance inflation as follows. The prior ensemble variance is inflated, multiplicatively, following $\mathbf{P}_{\text {ens }}^{f}=\rho \mathbf{P}_{\text {ens }}^{f}$. For each of the 10 iterations associated, we also iterate on prior multiplicative inflation values:

$$
\rho=\frac{\left\langle\left(\overline{\mathbf{x}_{b i}}-\mathbf{x}_{i}^{t}\right)^{2}\right\rangle}{\left\langle\sigma_{e}^{2}\right\rangle},
$$

where $\sigma_{e}^{2}$ is the ensemble variance, given by

$$
\sigma_{e}^{2}=\frac{1}{N_{e}-1} \sum_{i=1}^{N_{e}}\left(\mathbf{x}_{b i}-\overline{\mathbf{x}_{b}}\right)^{2} .
$$

We then adjust the inflation factor for the next iteration following

$$
\rho(i+1)=0.9 \times \rho(i-1)+0.1 \times \rho(i) .
$$

To prevent the inflation factor from adjusting too rapidly, we cap the overall change in inflation parameters at $10 \%$. Since the error statistics are sensitive to ensemble inflation, we write out a new $\mathbf{P}_{\text {clim }}^{f}$ on each iteration, such that the $\mathbf{P}_{\text {clim }}^{f}$ used for the hybridized experiments is obtained on the 10th iteration. Although the static error covariance matrix will generally be overvariant for hybridized cases, this is a more realistic model, given that NWP centers usually do not update their static covariance models when a hybrid system is used. We also acknowledge that the optimal linear combination may be one in which $w_{e}+w_{c} \neq 1$; however, for the sake of computational expense and modeling realistic tuning, we simply search for the $w_{e}$ value that gives the minimum posterior RMSE constrained to the one-dimensional search space by the condition $w_{e}+$ $w_{c}=1$.

3) We then perform standard hybrid integrations following (40) for each value of $w_{e}$ decreasing from 0.9 to 0 at 0.1 increments. This gives us 10 experiments for hybrid weighting $\left(w_{e}\right)$ values ranging from 1 to 0 . For each of these 10 experiments, run for 100000 time steps, we use the $100 \%$ - ensemble-based static $\mathbf{P}_{\text {clim }}^{f}$ but adjust the ensemble variances as described in (2), such that $\left\langle\sigma_{e}^{2}\right\rangle \approx \sigma_{f}^{2}$.

After applying steps 1-3, we have a set of experiments, for each alpha value, with a static climatological error covariance matrix (consistent with the $w_{e}=1$ case) and a flow-dependent error covariance matrix with appropriate dispersion. Figure 4 shows that for the $N_{e}=5$ case the inflated prior ensemble is near-optimally inflated, with the ratio falling along the $y=1$ line. The hybridized variance is perfect for the $100 \%$-ensemble case (since a $100 \%$-ensemble-based static is used) and overinflated when error variances are reduced through hybridization, where $0.2<w_{e}<1$. The posterior is undervariant for all cases in which the ensemble weighting is nonzero, where sampling error plays a role. For the $N_{e}=10$ case, the optimal performance is found when more weight is given to the ensemble. For $w_{e} \geq 0.5$, the prior and hybridized variances have a nearly optimal ratio (nearly equal to 1 ). For the larger ensemble size, sampling error is reduced and the posterior is only slightly undervariant.

We now turn our attention to deriving optimal static and flow-dependent weights in a computationally efficient manner. We perform a linear regression using the inflated ensemble variance as a predictor of the squared error. In this case, the truth is known, so we can compute the actual forecast error (even though we will have sampling error in the mean for these small ensemble sizes). In an operational setting, one would compute the forecast error variances following

$$
\sigma_{f}^{2}=\left\langle d^{2}\right\rangle-\sigma_{o}^{2},
$$

where $d$ denotes the debiased innovation $y^{o}-x^{f}$. The observation error variance $\sigma_{o}^{2}$ would need to be estimated using a method (e.g., Desroziers et al. 2005; Hollingsworth and Lönnberg 1986).

The slope term of the linear regression now gives a weight for the ensemble variance $w_{\text {ens }}$, and the weighting for the static term $w_{\text {clim }}$ is determined such that

$$
w_{\text {clim }}\left[\frac{\operatorname{tr}\left(\mathbf{P}_{\text {clim }}^{f}\right)}{M}\right]=g_{\text {clim }},
$$

where $\operatorname{tr}()$ denotes the trace of the matrix and $g_{\text {clim }}$ is the intercept term of the regression. We note that, from the definition in (47), $w_{\text {clim }}$ could act to inflate or attenuate the static covariances $\mathbf{P}_{\text {clim }}^{f}$, such that they equal the portion of the true error variance that does not vary as a function of ensemble variance. Figure 5 shows binned spread-skill diagrams with 10 bins. A perfectly dispersed ensemble would fall on the 1-to-1 line (solid red line); in such a case, we expect the optimal hybrid covariance to equal the ensemble-based covariance. If the ensemble has no skill, we expect the regression line to be horizontal, in which case the optimal hybrid covariance is purely static. We tend to see that the relationship between the ensemble variance and the error variance is curved rather than linear for this model, indicating that a higher-degree polynomial may provide a better fit. Figure $5 \mathrm{~d}$ shows the linear 

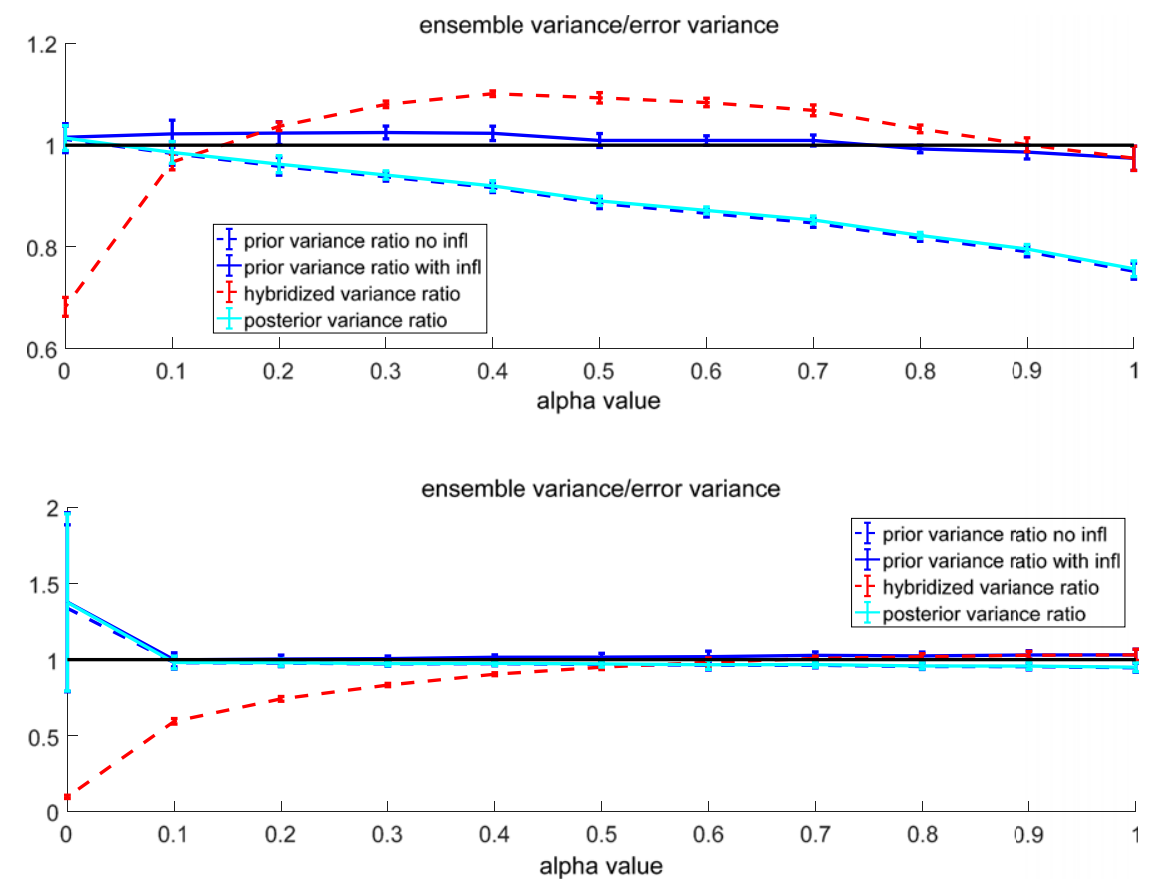

FIG. 4. The ratio of ensemble variance to error variance shown as a function of weight given to the ensemble variance in the hybrid system. Shown are the prior ratio (dashed blue line), inflated prior ratio (solid blue line), hybridized ratio (red dashed line), and posterior ratio (solid cyan line) for (top) $N_{e}=5$ and (bottom) $N_{e}=10$. Lines indicate the mean of iterations 4-9, and error bars show the $95 \%$ confidence interval. All experiments use an entirely ensemble based static.

regression-based hybridized variances, whereas Fig. 5e shows hybridized variances based on a cubic regression. The bottom panels of Fig. 5 show the $N_{e}=10$ member ensemble case. The 10-member ensemble case also indicated a curved spread-skill relationship; however, after several iterations, negative leading coefficients were found. This behavior indicates that the polynomial fit would produce decreasing variances as the ensemble variance became large, which could result in negative variances. In such cases, there are many ways to avoid decreasing or negative variances-for example, holding the fitted regression constant at the point that it begins to decrease (which is done in section 4d). However, for the sake of brevity, in this section we will simply show the linear model for the 10-member ensemble case. Figure 6 shows the ratio of ensemble variances to error variances by iteration for the $N_{e}=5$ linear regressionbased hybrid (top panel), $N_{e}=5$ cubic regression-based hybrid (middle panel), and $N_{e}=10$ linear regressionbased hybrid (bottom panel). Although there is some fluctuation in how quickly the optimal ratio is achieved, all experiments show prior ratios ranging between 0.8 and 1.1.

Figure 7 shows the RMSE as a function of ensemble weighting for $N_{e}=5$ (top) and $N_{e}=10$ (bottom). Also shown are the RMSEs based on regression. For the
$N_{e}=5$ brute-force tuning experiment, we get a minimum RMSE when $w_{e}=0.7$. The linear regression indicates $0.32<w_{e}<0.45$; therefore, because the spreadskill relationship is curved, the ensemble weights for a linear model are underestimated in this case. [We note that the magenta curve (linear regression) and the blue curve (tuning) are not equal for that range of weights since the $y$ intercept associated with the magenta curve is not forced to equal $\left(1-w_{e}\right) \mathbf{D}_{\text {clim }}$, as it would be if the static $\mathbf{P}_{\text {clim }}$ were the correct static for those ensemble weights.] The cubic regression-based hybrid shown in red gives a lower RMSE than any of the linear models, as would be expected based on the spread-skill plots shown in Fig. 5. For the $N_{e}=10$ case, the linear model also underestimates the optimal weights of $w_{e}=0.9$, which is again expected, from Fig. 5c.

\section{d. Impact of observation error, observing network density, and model error}

As indicated in the beginning of this section, one of the motivations in using a simplified model is the ability to control parameters such as the observation error variance $\sigma_{o}^{2}$, observation density, and model error. In the following, we investigate the impact of changes to these parameters on the results shown in section 4c. These 

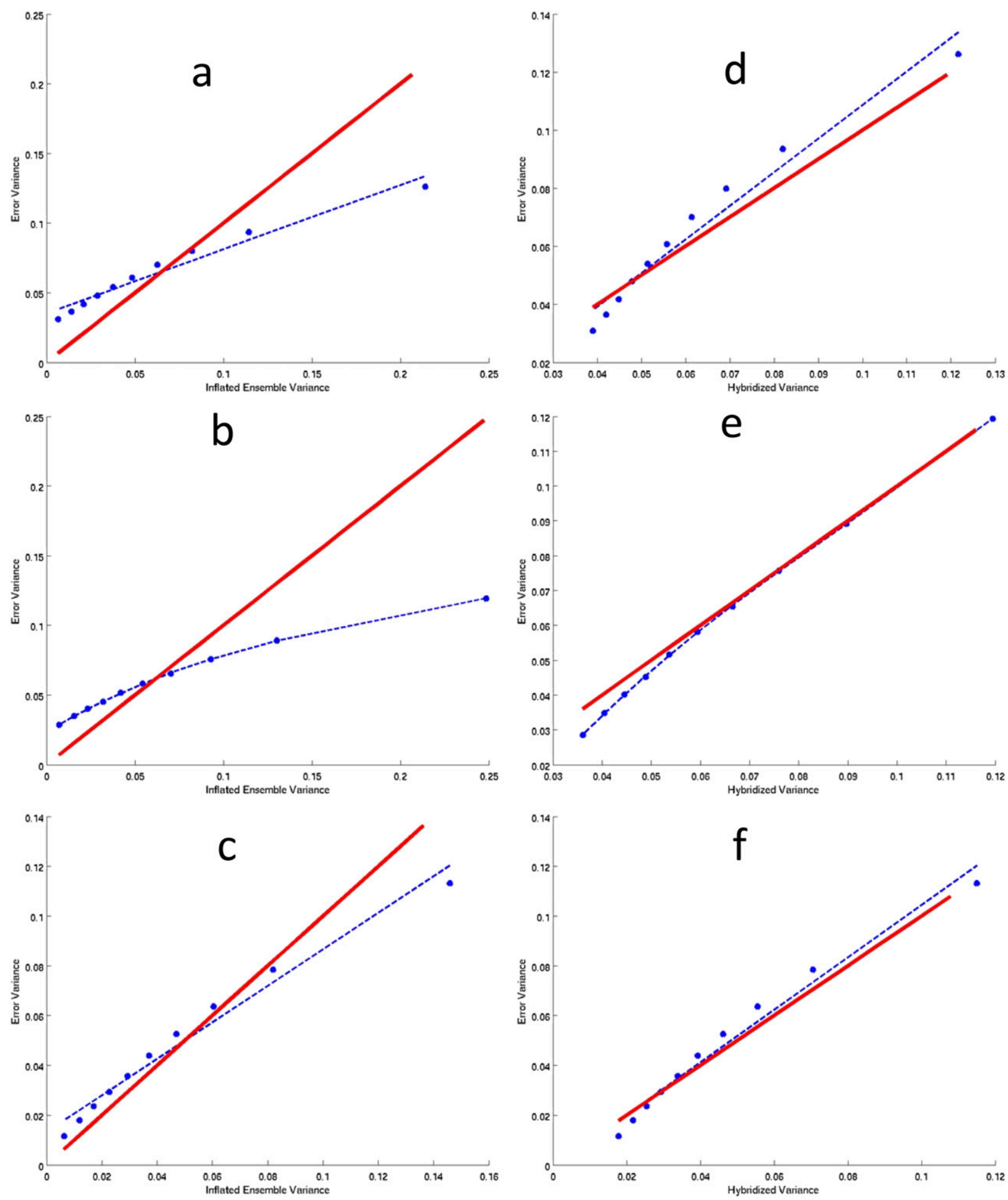

FIG. 5. Binned spread-skill diagrams for the (left) inflated prior ensemble and (right) hybridized ensemble for (a),(d) $N_{e}=5 \mathrm{member}$ ensemble with linear regression-based hybrid, (b),(e) $N_{e}=5$ member ensemble with cubic regression-based hybrid, and (c),(f) $N_{e}=10$ member ensemble with linear regression-based hybrid. The perfect 1-to-1 line is shown in red. The blue dots show the bin mean variances, and the dashed blue line shows the regression fit. 

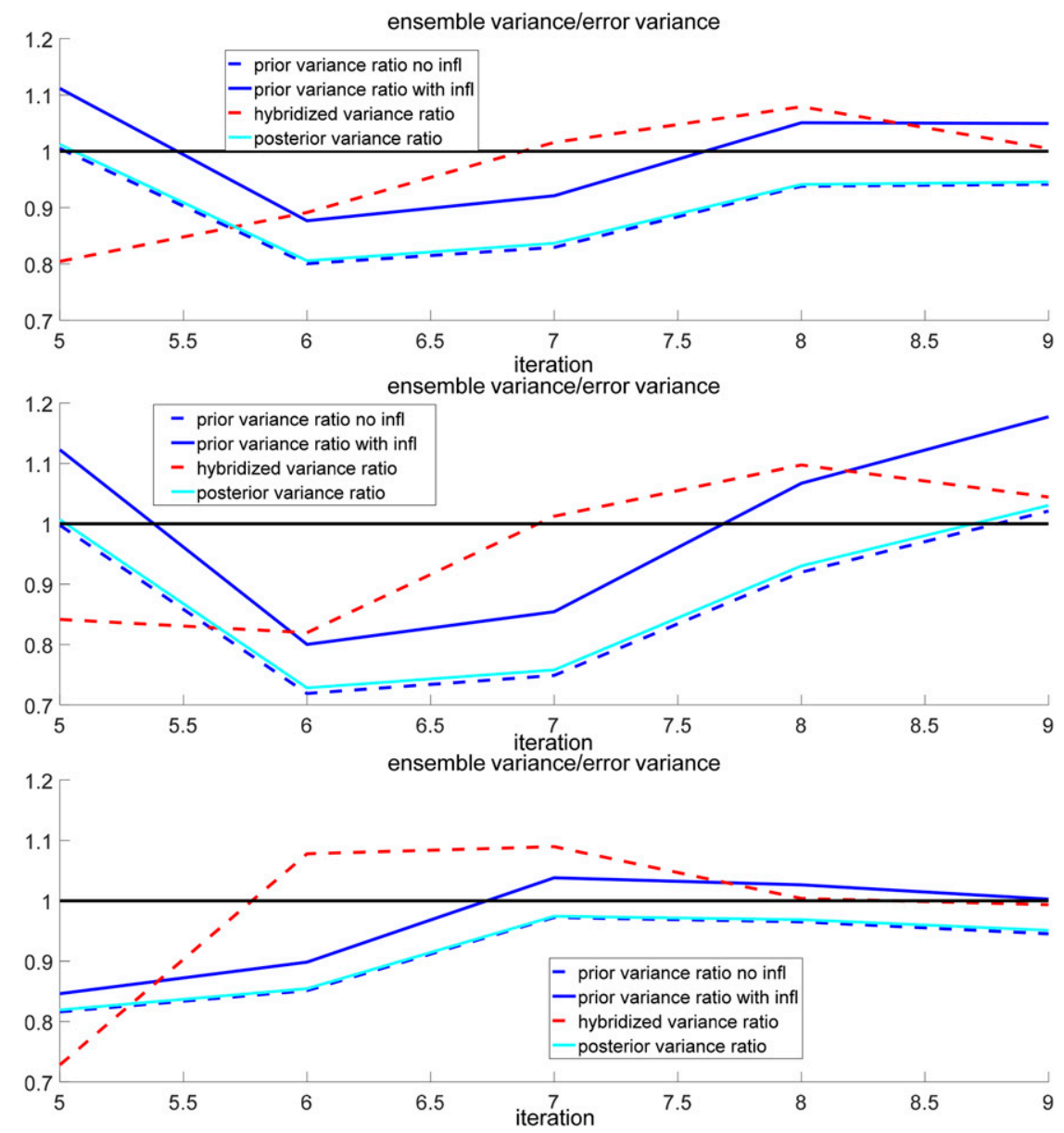

FIG. 6. The ratio of ensemble variance to error variance by iteration. Shown are the prior ratio (dashed blue line), inflated prior ratio (solid blue line), and hybridized ratio (red dashed line) for the (top) $N_{e}=5$ linear regression-based hybrid, (middle) $N_{e}=5$ cubic regressionbased hybrid, and (bottom) $N_{e}=10$ linear regression-based hybrid. All experiments use an entirely ensemble based static.

experiments help to test the limits of performance of these methods and to identify potential issues with implementation in an operational system. We proceed following the experiment design described in section $4 \mathrm{c}$, but here focus only on the $N_{e}=5$ case. In addition, since in many cases we are incorporating more severe errors, we found that we had to adjust the cubic fit to prevent decreasing with large ensemble values, as negative leading coefficients sometimes occurred. This was done by determining the maximum value of the function and holding that value constant as ensemble variance increased. Additionally, we set a lower bound to the cubic fit defined by the smaller of the minima of the inflated ensemble variance from the previous iteration or 0.0001 . The upper bound was defined by a $10 \%$ increase of the maximum of the fitted function from the previous iteration.

We first investigate the influence of changing the observation error. Figure 8 shows the RMSE as a function of ensemble weighting for the $N_{e}=5$ case. The top panel shows the case of observation error variance decreased by a factor of 100, and the bottom panel shows the case of the observation error variance being increased by a factor of 100. In this experiment, as in Fig. 7, all points are observed. In the top panel of Fig. 8, we see that although the error has been reduced by a factor of 10 , the optimal weighting of the ensemble found by brute-force tuning is very similar to that in Fig. 7. Here, the regression again shows that the spread-skill relationship is curved, and the cubic regression again shows improved performance when compared with the linear regression. When we increase the observation error variance by a factor of 100 (bottom panel), we find that the model background is less accurate and lower weight should be given to the ensemble. In this case, the minimum RMSE is found when the weight given to the ensemble is $w_{e}=0.4$. We also find very similar performance between the 

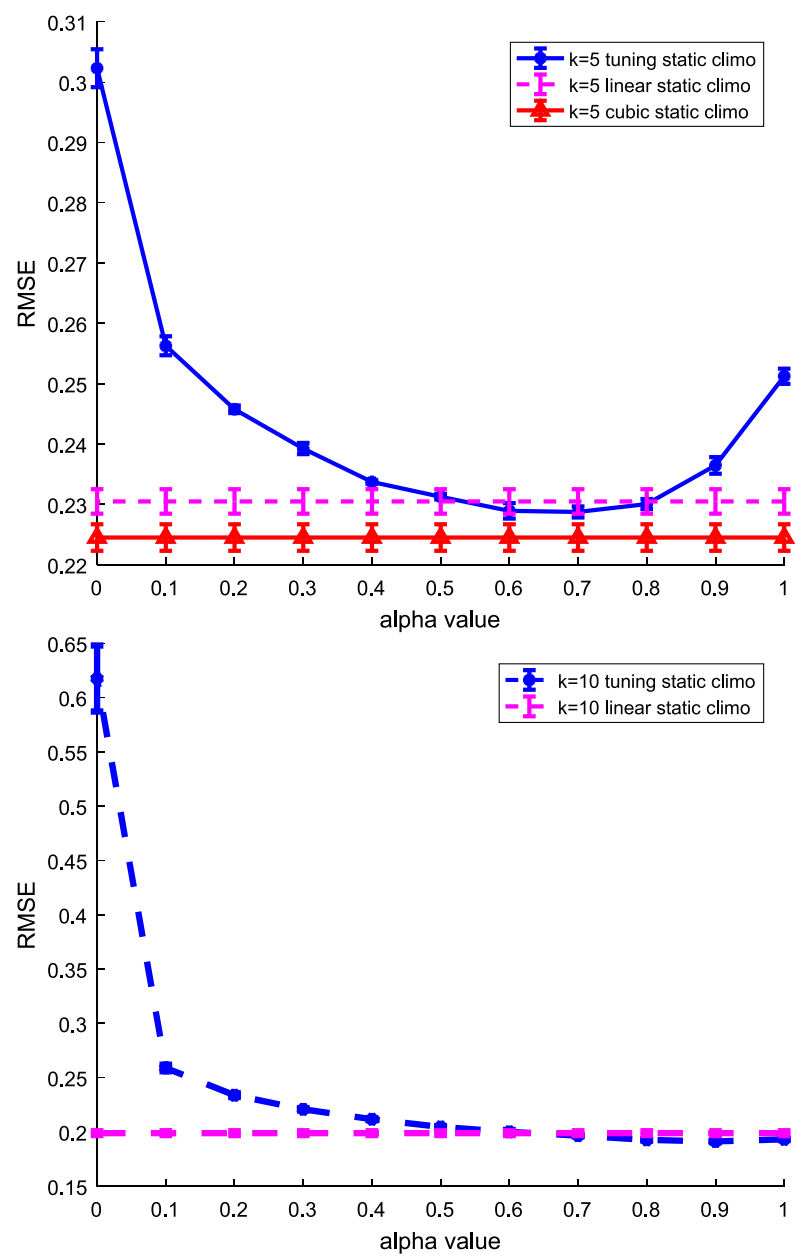

FIG. 7. RMSE as a function of ensemble weighting for (top) $N_{e}=5$ and (bottom) $N_{e}=10$. Also shown are the RMSE based on a linear regression (magenta) and cubic regression (red; shown only for $N_{e}=5$ ). Lines indicate the mean of iterations 4-9, and error bars show the $95 \%$ confidence interval. All experiments use an entirely ensemble based static.

cubic and linear regressions. This behavior is consistent with the spread-skill relationship becoming more horizontal, in the case of a less accurate ensemble.

We now investigate the impact of changing the observing density, but still defining $\sigma_{o}^{2}=0.5$ as in the baseline experiments shown in Fig. 7. We compare different observation densities, always observing the center points, so as to mimic observing differences over land versus ocean. Figure 9 shows RMSE as a function of ensemble weighting for the cases of $80 \%$ of the grid points observed (top) and $50 \%$ of the grid points observed. In these cases, $\mathbf{P}_{\text {clim }}^{f}$ has location dependence, so determining the weighting for the static term $w_{\text {clim }}$ on the basis of (47) is not consistent with brute-force tuning. Instead, we modify (47) to take the trace over only the observed points. In other words, we fit both the linear
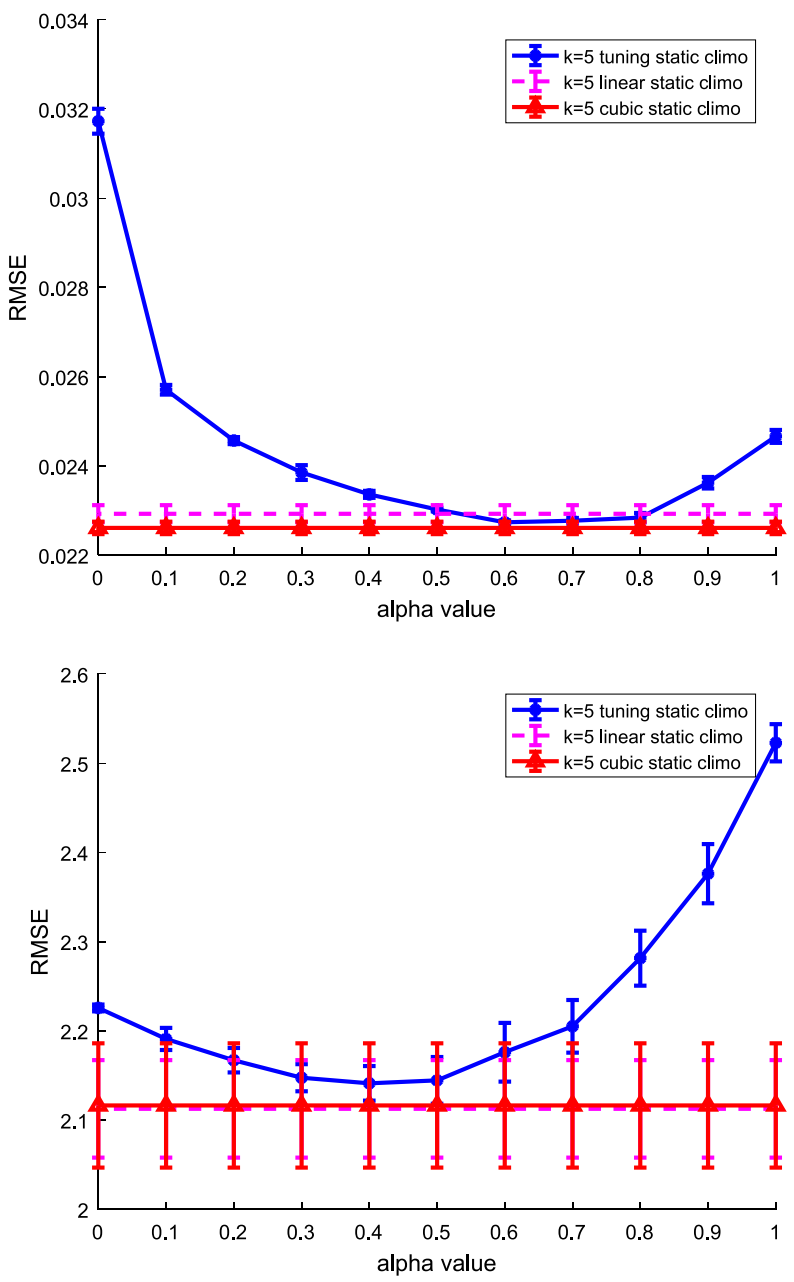

FIG. 8. RMSE as a function of ensemble weighting for $N_{e}=5$. Shown for observation error variance (top) decreased by a factor of 100 and (bottom) increased by a factor of 100 . Also shown are the RMSE based on a linear regression (magenta) and cubic regression (red). Lines indicate the mean of iterations 5-10, and error bars show the $95 \%$ confidence interval. All experiments use an entirely ensemble based static.

and cubic functions based on the observed locations and apply those functions at all locations, whether observed or unobserved. We note that in such cases improved performance could potentially be obtained by weighting unobserved points differently; however, this approach would require computationally expensive tuning and is outside the scope of this study. Figure 9 shows that when the observing density is reduced to $80 \%$ (top panel), we find the minimum posterior RMSE through brute-force tuning (computed over all grid points, whether observed or unobserved) $w_{e} \approx 0.8$. In this case, the ensemble and observations are still very accurate and the cubic fit offers some benefit over the linear fit. However, when the observation density is further reduced to $50 \%$, the linear 

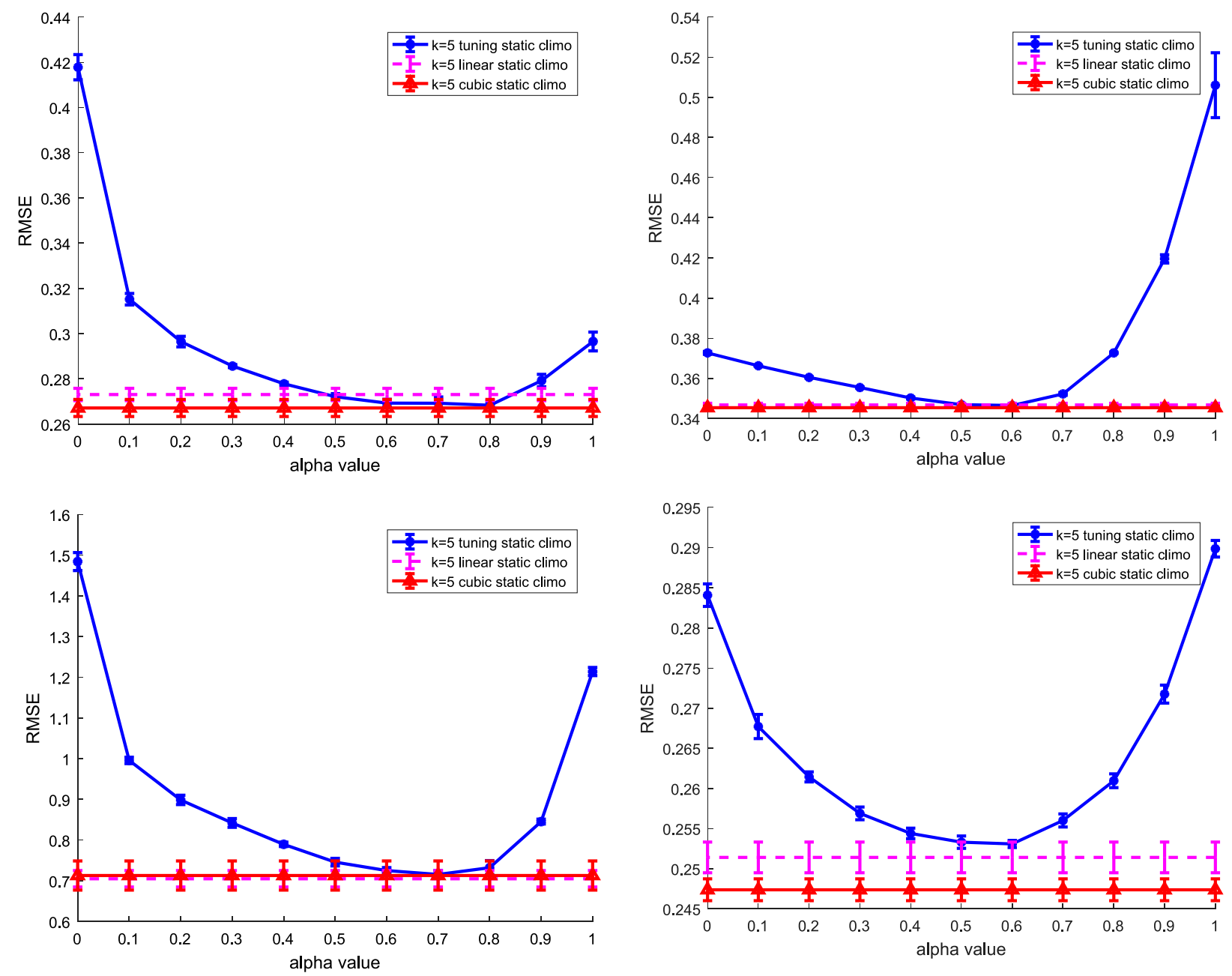

FIG. 9. As in Fig. 8, but for an observation density of (top) $80 \%$ and (bottom) $50 \%$. In both cases, the middle grid points are observed.

and cubic fits converge, with a minimum RMSE found when the ensemble weighting is $w_{e} \approx 0.7$.

Next, we turn our attention to the impact of model error. We simulate model error by forcing the nature run, which provides the true state for verification and is perturbed to create observations, to be determined by a different model. This simulation is achieved by changing the forcing term $F=10$ for the nature run but keeping the standard forcing $(F=8)$ for the experiment. The ensemble only accounts for this simulated model error through tuning multiplicative inflation. The results are shown in the top panel of Fig. 10. Again, consistent with a less accurate ensemble, the minimum RMSE found through brute-force tuning is achieved when the ensemble weighting is $w_{e} \approx 0.6$, and little difference is seen between the cubic and linear fits. Last, we consider state-dependent error by adding a model error with

FIG. 10. As in Fig. 8, but for (top) changing the forcing in the nature run from $f=8$ to $f=10$ and (bottom) including state-dependent model error (in this case Gaussian random noise with variance $q=0.05$ is added when ensemble states are greater than a threshold of 7).

variance drawn from a Gaussian with zero mean and variance $q=0.05$ ( $1 / 10$ of the observation error) to each ensemble member at each grid point where a value greater than a threshold of 7 is achieved (the typical range of values for this experimental setup is $[-15,15])$. In this case, the state-dependent model error changes both the ensemble mean and the variance. The RMSE is plotted as a function of weighting given to the ensemble covariance on the bottom panel of Fig. 10. Here, the optimal weighting given to the ensemble found through brute-force tuning is $w_{e} \approx 0.6$; however, the difference between the cubic and linear fits is still significant.

In all of the cases considered in this section, the cubic fit has either equally performed or outperformed the optimal weights found through brute-force tuning. It is also found that the degree to which the cubic outperforms the best linear model is based on the curvature of the 
spread-skill relationship, which depends on the performance of the ensemble. While the above results indicated that fitting to a higher-degree polynomial is most important when the ensemble variance more accurately tracks the true error variance, Fig. 5 indicated that, as the ensemble size increases, the spread-skill relationship becomes more linear. These two results together indicate that there may be a range in which a higher-order polynomial fit is preferable. It is also worth noting that, in many of the cases in which the cubic outperforms the linear regression, there is a more optimal linear fit, shown through brute-force tuning, than that estimated by regression.

\section{Conclusions}

In this study, we built on the findings of Bishop and Satterfield (2013) to show that the expected true prior variance, given an ensemble sample variance, is consistent with linear regression for the case in which the climatological distribution of true error variances is described by an inverse gamma; the distribution of ensemble variances, given a true error variance, is a gamma PDF; and the distribution of the state, given a true forecast error variance, is Gaussian. Theory was developed to confirm that, when only the sampling error in the ensemble variance was considered, the gain that minimizes the posterior error variance uses the expected true prior variance, given an ensemble sample variance. We demonstrated that, after a single run of a fully ensemble data assimilation scheme, one can use regression to obtain a model of optimal hybrid variance. For the idealized univariate data assimilation and multivariate cycling ensemble data assimilation considered here, it was found that, when the relationship between the ensemble variance and true error variance is linear, linear regression closely approximates the optimal weights found through the simple, but computationally expensive, process of testing every plausible combination of weights. For the case in which the relationship between the ensemble variance and true error variance is nonlinear, we introduce a generalized hybrid model defined by higher-order polynomial regression and demonstrated that such a scheme outperformed any plausible linear hybrid model. It is also found that the degree to which higher-order polynomial regression outperforms the best linear model is dependent on the performance of the ensemble. Typically, fitting to a higher-degree polynomial is most important when the ensemble variance more accurately tracks the true error variance.

The focus of this work has been on hybrid formulations that form a hybridized error covariance matrix (e.g., Hamill and Snyder 2000) and for filters that assimilate all available observations at once. Additional work would be needed to implement serial filters. The theory presented here has only been applied to variances, and additional work is needed to account for correlations, although a similar method could be applied. In addition, it would be of interest to examine how to optimally form a static error covariance matrix and update such a matrix with updates to hybrid coefficients. These issues, as well as application of this theory to the Navy Global Environmental Model (NAVGEM), are the subject of future work.

Acknowledgments. The authors thank the three anonymous reviewers, whose suggestions helped us to improve the presentation of our results. This research is supported by the Office of Naval Research (ONR) through the NRL Base Program PE 0601153N.

\section{APPENDIX}

\section{Derivation of the Kalman Gain}

We follow the assumptions of section $2 b$, such that we write the true posterior mean in this case as

$$
\begin{aligned}
& \bar{x}_{a}=\bar{x}+G_{t}(y-\bar{x}), \text { with } \\
& G_{t}=\frac{\sigma_{t}^{2}}{\sigma_{t}^{2}+\sigma_{o}^{2}},
\end{aligned}
$$

and the one with sampling error will be written as

$$
\bar{x}_{e}=\bar{x}+G_{e}(y-\bar{x}) .
$$

We emphasize that, consistent with section $2 b$, we have assumed for simplicity that the only sampling error comes in through the gain and the prior variance. Note that we have not assumed a form for $G_{e}$; it has been left completely arbitrary. It is the goal of this section to derive its optimal representation.

We perform the standard steps to minimize the posterior variance. The posterior variance about (A3) in this case is

$$
P_{a}=\int_{0}^{\infty} \int_{-\infty}^{\infty} \int_{-\infty}^{\infty}\left(x-\bar{x}_{e}\right)^{2} p\left(\sigma_{t}^{2} \mid \sigma_{e}^{2}\right) p\left(y \mid \sigma_{t}^{2}, \sigma_{e}^{2}\right) p\left(x \mid y, \sigma_{t}^{2}, \sigma_{e}^{2}\right) d x d y d \sigma_{t}^{2} .
$$


Using (A1)-(A3) in (A4), we obtain

$$
\begin{aligned}
P_{a}= & \int_{-\infty}^{\infty} P_{t} p\left(\sigma_{t}^{2} \mid \sigma_{e}^{2}\right) d \sigma_{t}^{2} \\
& +\int_{-\infty}^{\infty}\left(G_{t}-G_{e}\right)^{2}\left(\sigma_{t}^{2}+\sigma_{o}^{2}\right) p\left(\sigma_{t}^{2} \mid \sigma_{e}^{2}\right) d \sigma_{t}^{2},
\end{aligned}
$$

where the true posterior variance is $P_{t}=\sigma_{t}^{2}-G_{t} \sigma_{t}^{2}$. The first term on the right-hand side is the true posterior error variance averaged over the climatological distribution of true variances. Let us call this $\left\langle P_{t} \mid \sigma_{e}^{2}\right\rangle$, and therefore we have

$$
P_{a}=\left\langle P_{t} \mid \sigma_{e}^{2}\right\rangle+\int_{-\infty}^{\infty}\left(G_{t}-G_{e}\right)^{2}\left(\sigma_{t}^{2}+\sigma_{o}^{2}\right) p\left(\sigma_{t}^{2} \mid \sigma_{e}^{2}\right) d \sigma_{t}^{2} .
$$

The integral on the right decomposes as

$$
\begin{aligned}
\int_{-\infty}^{\infty} & \left(G_{t}-G_{e}\right)^{2}\left(\sigma_{t}^{2}+R\right) p\left(\sigma_{t}^{2} \mid \sigma_{e}^{2}\right) d \sigma_{t}^{2} \\
= & \left\langle\sigma_{t}^{2} \mid \sigma_{e}^{2}\right\rangle-\left\langle P_{t} \mid \sigma_{e}^{2}\right\rangle-2\left\langle\sigma_{t}^{2} \mid \sigma_{e}^{2}\right\rangle G_{e} \\
& +G_{e}^{2}\left(\left\langle\sigma_{t}^{2} \mid \sigma_{e}^{2}\right\rangle+\sigma_{o}^{2}\right) .
\end{aligned}
$$

Using (A7) in (A6), we obtain

$$
P_{a}=\left\langle\sigma_{t}^{2} \mid \sigma_{e}^{2}\right\rangle-2\left\langle\sigma_{t}^{2} \mid \sigma_{e}^{2}\right\rangle G_{e}+G_{e}^{2}\left(\left\langle\sigma_{t}^{2} \mid \sigma_{e}^{2}\right\rangle+\sigma_{o}^{2}\right) .
$$

Next, we take the derivative of (A8) with respect to $G_{e}$ and set the result to zero:

$$
\frac{d P_{a}}{d G_{e}}=-2\left[\left\langle\sigma_{t}^{2} \mid \sigma_{e}^{2}\right\rangle-G_{e}\left(\left\langle\sigma_{t}^{2} \mid \sigma_{e}^{2}\right\rangle+\sigma_{o}^{2}\right)\right]=0 .
$$

We then solve (A9) for the sought-after result, namely,

$$
G_{e}=\frac{\left\langle\sigma_{t}^{2} \mid \sigma_{e}^{2}\right\rangle}{\left\langle\sigma_{t}^{2} \mid \sigma_{e}^{2}\right\rangle+\sigma_{o}^{2}} .
$$

\section{REFERENCES}

Anderson, J. L., 2007: Exploring the need for localization in ensemble data assimilation using a hierarchical ensemble filter. Physica D, 230, 99-111, https://doi.org/10.1016/ j.physd.2006.02.011.

Bishop, C. H., and E. A. Satterfield, 2013: Hidden error variance theory. Part I: Exposition and analytic model. Mon. Wea. Rev., 141, 1454-1468, https://doi.org/10.1175/MWR-D-12-00118.1.

— - _ and K. T. Shanley, 2013: Hidden error variance theory. Part II: An instrument that reveals hidden error variance distributions from ensemble forecasts and observations. Mon. Wea. Rev., 141, 1469-1483, https://doi.org/10.1175/MWR-D-12-00119.1.

Bocquet, M., 2011: Ensemble Kalman filtering without the intrinsic need for inflation. Nonlinear Processes Geophys., 18, 735-750, https://doi.org/10.5194/npg-18-735-2011.

Burgers, G., P. Jan van Leeuwen, and G. Evensen, 1998: Analysis scheme in the ensemble Kalman filter. Mon. Wea. Rev., 126, 1719-1724, https://doi.org/10.1175/1520-0493(1998)126<1719: ASITEK $>2.0 . \mathrm{CO} ; 2$.
Clayton, A. M., A. C. Lorenc, and D. M. Barker, 2013: Operational implementation of a hybrid ensemble/4D-Var global data assimilation system at the Met Office. Quart. J. Roy. Meteor. Soc., 139, 1445-1461, https://doi.org/10.1002/qj.2054.

Desroziers, G., L. Berre, B. Chapnik, and P. Poli, 2005: Diagnosis of observation, background and analysis-error statistics in observation space. Quart. J. Roy. Meteor. Soc., 131, 3385-3396, https://doi.org/10.1256/qj.05.108.

Etherton, B. J., and C. H. Bishop, 2004: Resilience of hybrid ensemble/3DVAR analysis schemes to model error and ensemble covariance error. Mon. Wea. Rev., 132, 1065-1080, https://doi.org/10.1175/1520-0493(2004)132<1065:ROHDAS> 2.0.CO;2.

Furrer, R., and T. Bengtsson, 2007: Estimation of high-dimensional prior and posterior covariance matrices in Kalman filter variants. J. Multivar. Anal., 98, 227-255, https://doi.org/10.1016/ j.jmva.2006.08.003.

Gaspari, G., and S. E. Cohn, 1999: Construction of correlation functions in two and three dimensions. Quart. J. Roy. Meteor. Soc., 125, 723-757, https://doi.org/10.1002/qj.49712555417.

Hamill, T. M., and C. Snyder, 2000: A hybrid ensemble Kalman filter-3D variational analysis scheme. Mon. Wea. Rev., 128, 2905-2919, https://doi.org/10.1175/1520-0493(2000)128<2905: AHEKFV $>2.0 . \mathrm{CO} ; 2$.

— J. S. Whitaker, and C. Snyder, 2001: Distance-dependent filtering of background error covariance estimates in an ensemble Kalman filter. Mon. Wea. Rev., 129, 2776-2790, https:/doi.org/ 10.1175/1520-0493(2001)129<2776:DDFOBE>2.0.CO;2.

Hodyss, D., W. F. Campbell, and J. S. Whitaker, 2016: Observation-dependent posterior inflation for the ensemble Kalman filter. Mon. Wea. Rev., 144, 2667-2684, https://doi.org/10.1175/ MWR-D-15-0329.1.

Hollingsworth, A., and P. Lönnberg, 1986: The statistical structure of short-range forecast errors as determined from radiosonde data. Part I: The wind field. Tellus, 38A, 111-136, https:// doi.org/10.1111/j.1600-0870.1986.tb00460.x.

Kuhl, D. D., T. E. Rosmond, C. H. Bishop, J. McLay, and N. L. Baker, 2013: Comparison of hybrid ensemble/4DVar and 4DVar within the NAVDAS-AR data assimilation framework. Mon. Wea. Rev., 141, 2740-2758, https://doi.org/10.1175/ MWR-D-12-00182.1.

Lorenc, A. C., 2003: The potential of the ensemble Kalman filter for NWP-A comparison with 4D-Var. Quart. J. Roy. Meteor. Soc., 129, 3183-3203, https://doi.org/10.1256/qj.02.132.

Lorenz, E. N., 1996: Predictability: A problem solved. Proc. Seminar on Predictability, Shinfield Park, Reading, United Kingdom, ECMWF, 18 pp.

, 2005: Designing chaotic models. J. Atmos. Sci., 62, 1574-1587, https://doi.org/10.1175/JAS3430.1. 
Ménétrier, B., and T. Auligné, 2015: Optimized localization and hybridization to filter ensemble-based covariances. Mon. Wea. Rev., 143, 3931-3947, https://doi.org/10.1175/MWRD-15-0057.1.

, T. Montmerle, Y. Michel, and L. Berre, 2015: Linear filtering of sample covariances for ensemble-based data assimilation. Part I: Optimality criteria and application to variance filtering and covariance localization. Mon. Wea. Rev., 143, 1622-1643, https://doi.org/10.1175/MWR-D14-00157.1.

Sacher, W., and P. Bartello, 2008: Sampling errors in ensemble Kalman filtering. Part I: Theory. Mon. Wea. Rev., 136, 30353049, https://doi.org/10.1175/2007MWR2323.1.

Wang, X., D. Barker, C. Snyder, and T. M. Hamill, 2008a: A hybrid ETKF-3DVAR data assimilation scheme for the WRF
Model. Part I: Observing system simulation experiment. Mon. Wea. Rev., 136, 5116-5131, https://doi.org/10.1175/ 2008MWR2444.1.

,,,--- and $-2008 \mathrm{~b}$ : A hybrid ETKF-3DVAR data assimilation scheme for the WRF Model. Part II: Real observation experiments. Mon. Wea. Rev., 136, 5132-5147, https://doi.org/ 10.1175/2008MWR2445.1.

— D. Darrish, D. Kleist, and J. Whitaker, 2013: GSI 3DVar-based ensemble-variational hybrid data assimilation for NCEP Global Forecast System: Single-resolution experiments. Mon. Wea. Rev., 141, 4098-4117, https://doi.org/10.1175/MWR-D-12-00141.1.

Yaremchuk, M., D. Nechaev, and C. Pan, 2011: A hybrid background error covariance model for assimilating glider data into a coastal ocean model. Mon. Wea. Rev., 139, 1879-1890, https://doi.org/10.1175/2011MWR3510.1. 